\title{
Morfometría de los Nervios Isquiático, Tibial y Fibular Común en el Hombre
}

\author{
Morphometry of the Sciatic, Tibial and Common Fibular Nerves in Man
}

\author{
"Mariano del Sol; "Enrique Olave; *Jaime Contreras \& **Bélgica Vásquez
}

DEL SOL, M.; OLAVE, E.; CONTRERAS, J. \& VÁSQUEZ, B. Morfometría de los nervios isquiático, tibial y fibular común en el Hombre. Int. J. Morphol., 28(2):385-388, 2010.

RESUMEN: El nervio isquiático, el más extenso del cuerpo humano, emerge de la pelvis por el foramen isquiático mayor, dividiéndose proximal a la articulación de la rodilla en los nervios tibial y fibular común. Con frecuencia, estos nervios son afectados por accidentes, por tanto, la microcirugía reparadora requiere de detallada información anatómica para efectuar con éxito sus procedimientos. El nervio isquiático puede ser lesionado por heridas penetrantes, en las luxaciones posteriores de la articulación coxal y por inyecciones intramusculares mal aplicadas en la región glútea, siendo rara la lesión del nervio tibial por estar situado profundamente protegido. En cambio, la lesión del nervio fibular común, es frecuente debido a su posición superficial, quedando muy expuesto cuando abandona la fosa poplítea y rodea el cuello de la fíbula. Realizamos el estudio debido a la escasa información morfométrica y estereológica de estos nervios. Se disecaron en 5 cadáveres de individuos adultos, de sexo masculino los nervios isquiático, tibial y fibular común. Se obtuvieron secciones transversales de cada uno de los nervios a nivel de la división del nervio isquiático y del origen de los nervios tibial y fibular común. Realizamos cortes de $5 \mu \mathrm{m}$ de grosor, los que fueron teñidos con H.E. Obtuvimos información cuantitativa sobre medidas morfométricas, determinamos el número de fibras nerviosas de cada fascículo así como el número de fascículos existente en cada nervio. Además, determinamos, por planimetría a través del conteo de puntos, el área de los fascículos que constituían cada nervio. El número de fascículos en el nervio isquiático varió de 63 a 70, con un promedio de 66,8 (D. E. 2,59). En el nervio tibial, el número de fascículos varió de 35 a 43, con un promedio de 30 (D. E. 3,00) y en el nervio fibular común el número de fascículos varió de 17 a 25 , con un promedio de 21 (D. E. 2,92). El número promedio de fibras en el nervio ciático fue de 64.535 (D. E. 3.193). En los nervios tibial fue 40.317 (D. E. 4.067) y en el nervio fibular común fue 22.191 (D.E. 1.038). El área promedio de los nervios isquiático, tibial y fibular común fue: $11,42 \mathrm{~mm}^{2}$ (D. E. 0,54), 8,27 $\mathrm{mm}^{2}$ (D. E. 0,69) y $3,71 \mathrm{~mm}^{2}$ (D. E. 0,30), respectivamente. A través de este estudio esperamos contribuir al conocimiento morfométrico de estos nervios, sirviendo de apoyo tanto anatómico como quirúrgico.

PALABRAS CLAVE: Nervio isquiático; Nervio tibial; Nervio fibular común; Morfometría.

\section{INTRODUCCIÓN}

El nervio isquiático es el más largo del cuerpo humano, deja la pelvis a través del foramen isquiático mayor, abajo del músculo piriforme se curva lateral e inferiormente a través de la región glútea. Superiomente, se sitúa profundo al músculo glúteo máximo, cruza posteriormente a los músculos obturador interno, gemelos y cuadrado femoral quien lo separa del músculo obturador externo y articulación coxal, encontrándose situado entre la espina iliaca postero superior y el túber isquiático y luego entre el vértice del trocánter mayor y el túber isquiático. A seguir, el nervio isquiático pasa hacia distal, en la línea mediana (punto mediano entre el túber isquiático y el trocánter mayor hasta el vértice de la fosa poplítea) en la cara posterior del muslo, situándose posterior al músculo aductor magno, siendo cruzado por la cabezza larga del músculo bíceps femoral y se divide en los nervios tibial y fibular común en un lugar variable proximal a la fosa poplítea.

Según Williams et al. (1995) el nervio tibial es la mayor división del nervio isquiático, proveniente del IV y $\mathrm{V}$ nervios lumbares y del $1^{\circ}$ al $3^{\circ}$ nervios sacrales y el nervio fibular común es, aproximadamente, la mitad del tamaño del nervio tibial y derivado del IV y $\mathrm{V}$ nervios lumbares y del $1^{\circ}$ al $2^{\circ}$ nervios sacrales.

\footnotetext{
* Facultad de Medicina, Universidad de La Frontera, Temuco, Chile.

** Universidad Autónoma de Chile, Sede Temuco, Chile.

Financiado por DIUFRO, Proyecto N ${ }^{\circ}$ D106-0004
} 
Conocer el número de fibras de un nervio y de cada uno de los ramos en que se subdivide, adquiere importancia en los procedimientos utilizados en las estimulaciones con propósitos terapéuticos.

Por otra parte, la determinación del número de fibras en cada nervio, permitirá dilucidar cuál de los ramos de división aporta mayor cantidad de fibras, el nervio tibial o el nervio fabular común, de tal modo que se podrían conocer mejor las áreas de distribución en aquellos músculos con mayor o menor inervación.

Sabemos que la microcirugía reparadora necesita de abundante y fina información anatómica, esperamos que, con los datos obtenidos en este estudio, contribuir con nuevos antecedentes que podrían ser utilizados en los diversas intervenciones en la región posterior del muslo y de la pierna.

Llano et al. (2008) en un estudio realizado en perros, identificaron el número y situación espacial de los fascículos que conforman los nervios tibial y fibular común. Estos autores identificaron 13 fascículos en el nervio tibial y sólo 3 en el nervio fibular común.

En un estudio previo del Sol et al. (2005) determinaron que el número de fascículos en el nervio tibial, próximo a su división en los nervios plantares, variaba entre 19 y 39 , con un promedio de 28,6 (D.S. 7.13). Además, señalaron que el área promedio de los fascículos en los nervios tibial, plantar medial y plantar lateral fue de $3,66 \mathrm{~mm}^{2}, 1,8 \mathrm{~mm}^{2} \mathrm{y}$ $1,48 \mathrm{~mm}^{2}$, respectivamente.

Existe escasa información morfométrica de los nervios isquiático, tibial y fibular profundo en el Hombre, especialmente la conformación fascicular y el número de fibras intraneurales que los constituyen, hecho que nos motivó a estudiar algunos parámetros morfométricos y contribuir de esta manera, al conocimiento anatómico de estos nervios del miembro inferior.

\section{MATERIAL Y MÉTODO}

Se estudiaron 5 miembros inferiores de cadáveres adultos, de sexo masculino, provenientes de la Facultad de Medicina de la Universidad de La Frontera, Temuco, Chile. Se obtuvieron 5 secciones transversales de 5 nervios isquiático, tibial y fibular común. Las secciones fueron procesadas para su inclusión en paraplast. Realizamos cortes de $5 \mu \mathrm{m}$ de grosor, los que fueron montados en portaobjetos y teñidos con hematoxilina-eosina.

Determinamos el número de fibras nerviosas de cada fascículo y el número de fascículos existente en cada nervio. Además, se calculó el área de los fascículos que constituían cada nervio, por planimetría a través del conteo de puntos.

Los cortes fueron fotografiados utilizando un microscopio Carl Zeiss, Axiolab, con cámara MC 80 DX. Para la morfometría utilizamos un microscopio Olympus con retículo ocular $(1 \mathrm{~mm} / 0,01 \mathrm{~mm})$ calibrándolo con un retículo objetivo (Mandarim-de-Lacerda, 1995).

\section{RESULTADOS}

En el nervio isquiático de los 5 miembros inferiores, el número de fascículos varió de 63 a 70, con un promedio de 66,8 (D.E. 2,59). En los 5 nervios tibiales el número de fascículos varió de 35 a 43, con un promedio de 30 (D.E. $3,00)$ y en el nervio fibular común el número de fascículos varió de 17 a 25 , con un promedio de 21 (D.E. 2,92). El número de fibras totales de cada nervio se presenta en la Fig. 1.

En promedio, el número de fibras que comprende el nervio ciático fue de 64.535 (D.E. 3.193). En los nervios

Tabla I. Suma de las áreas de los fascículos de los nervios isquiático, tibial y fibular común.

\begin{tabular}{cccc}
\hline Muestra & N. isquiático $\left(\right.$ área $\left.\mathrm{mm}^{2}\right)$ & N. tibial $\left(\right.$ área $\left.\mathrm{mm}^{2}\right)$ & N. fibular común $\left(\right.$ área $\left.\mathrm{mm}^{2}\right)$ \\
\hline 1 & 11,43 & 8,04 & 3,90 \\
2 & 12,00 & 8,82 & 4,01 \\
3 & 11,67 & 8,85 & 3,72 \\
4 & 11,48 & 8,44 & 3,67 \\
5 & 10,55 & 7,18 & 3,23 \\
\hline Promedio & 11,42 & 8,27 & 3,71 \\
D. E. & 0,54 & 0,69 & 0,30 \\
\hline
\end{tabular}




\section{N. isquiático $\square$ N. tibial $\square$ N. fibular común}

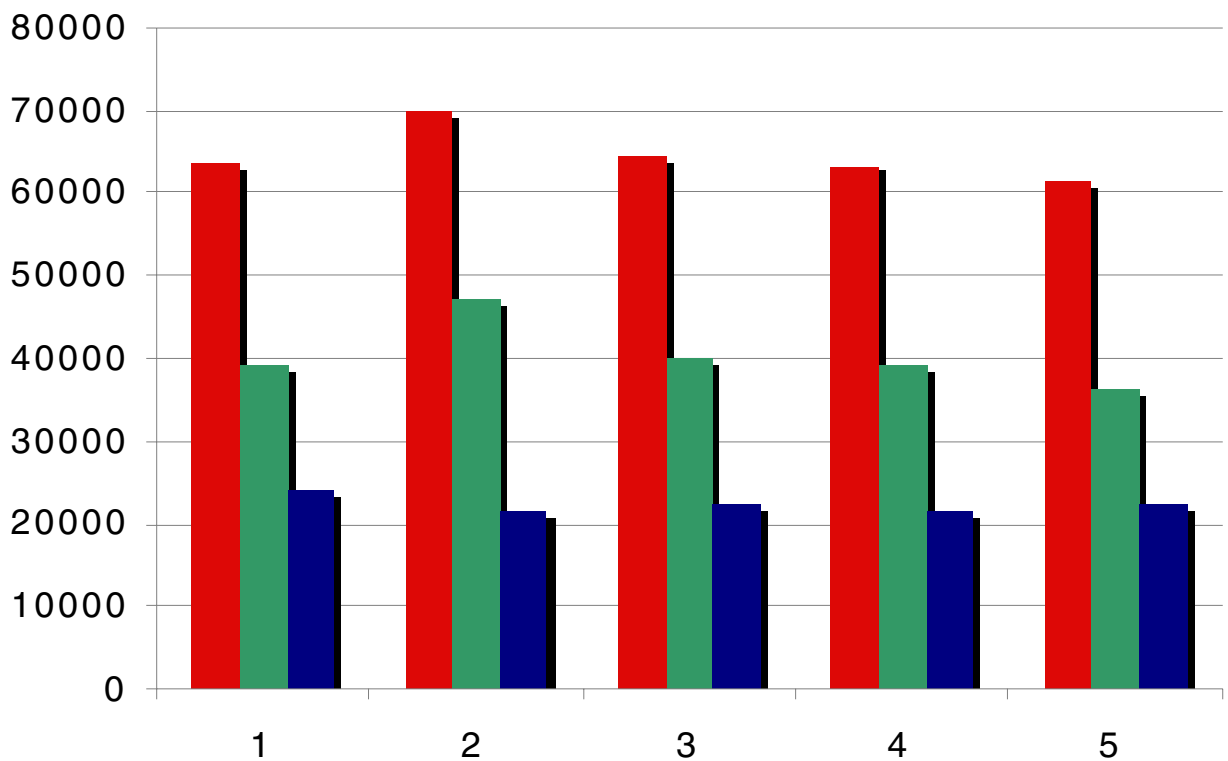

Fig. 1. Número de fibras en cada uno de los nervios isquiático, tibial y fibular común.

tibial fue de 40.317 (D.E. 4.067) y en el nervio fibular común fue de 22.191 (D.E. 1.038). En la Tabla I se muestra la suma de las áreas de los fascículos que constituyen los nervios isquiático, tibial y fibular común.

El área promedio de los nervios isquiático, tibial y fibular común fue: $11,42 \mathrm{~mm}^{2}$ (D. E. 0,54$), 8,27 \mathrm{~mm}^{2}$ (D. E. $0,69)$ y $3,71 \mathrm{~mm}^{2}$ (D. E. 0,30 ), respectivamente (Tabla I).

\section{DISCUSIÓN}

En los texto de Anatomía clásica, en general, se describe bien los trayectos y relaciones de los nervios espinales (Testut \& Jacob, 1978; Testut \& Latarjet, 1969; Orts, 1982; Williams et al. y Moore \& Dalley, 2002); sin embargo, en casi la totalidad no se detallan, aspectos mesoscópicos, morfométricos y estereológicos sobre los nervios isquiático, tibial y fibular común. Existen algunos estudios morfométricos de los nervios plantares medial y lateral ramos terminales del nervio tibial (del Sol et al., 2005) y de los ramos del nervio plantar lateral, desde su origen hasta su división en ramos superficial y profundo (del Sol et al., 2006)

Uno de los factores que puede incidir en la morfometría de estos nervios, corresponde a las eventuales variaciones musculares. Se sabe que la mayor cantidad de fibras que llevan estos nervios son de origen motor y están destinadas a los músculos de la región posterior del muslo, la pierna y el pie. Por lo tanto, al existir estas variaciones, el número de fascículos y de fibras nerviosas también van a ser variables, siendo superior en el nervio isquiático cuando de éste se originan los dos nervios tibial y fibular común. Se postula también, que pueden existir variaciones significativas en el número de fascículos en el nervio tibial por el gran territorio motor, especialmente en la región posterior de la pierna y en la musculatura del pie, a excepción del músculo extensor corto de los dedos del pie. A pesar de lo anteriormente señalado, no se observó una variación importante en el nervio tibial 35 a 43 fascículos como en el nevio fibular común 17 a 25 fascículos.

El nervio tibial destina a la parte posterior de la pierna, aproximadamente 10.000 fibras en promedio, ya que a nivel de la bifurcación del nervio isquiático, según los resultados obtenidos, presenta en promedio 40.317 fibras, y a nivel de la bifurcación en los nervios plantares, 31.783 fibras en promedio, según los estudios de del Sol et al. (2006).

El área promedio de los fascículos en el nervio tibial, inmediatamente después de la división del nervio isquiático, fue de $8,27 \mathrm{~mm}^{2}$, es decir, aproximadamente el doble del área que ocupa el mismo nervio, antes de su bifurcación en los nervios plantares $\left(3,66 \mathrm{~mm}^{2}\right)$, lo que concuerdo con el estudio de del Sol et al. (2005). Esta disminución en el área del nervio tibial se debe, sin lugar a dudas, a los numerosos ramos motores que emite durante su trayecto en la región posterior de la pierna. 
Al comparar las áreas de los nervios tibial y fibular común $\left(8,27 \mathrm{~mm}^{2}\right.$ versus $\left.3,71 \mathrm{~mm}^{2}\right)$ observamos que son semejantes a la aseveración de Williams et al., quienes señalaron que el nervio fibular común presenta, aproximadamente la mitad del tamaño del nervio tibial.

El nervio isquiático cuando es lesionado por inyecciones intramusculares mal aplicadas en la región glútea, se ven afectadas, casi siempre, las fibras que componen el nervio fibular común, (a pesar de ser la mitad tamaño del nervio tibial), debiéndose probablemente al hecho que las fibras del nervio fibular común se sitúan más superficialmente en el nervio isquiático. Si hay lesión en el tronco del nervio fibular común se pierde totalmente la extensión de los dedos y la dorsiflexión del tobillo.

Finalmente, cabe resaltar la ausencia de trabajos específicos sobre morfometría y estereología de los nervios periféricos. Conocer aspectos como diámetro de los nervios, perímetro de cada uno de ellos, número de fibras nerviosas, áreas de los fascículos y ramos nerviosos entre otros parámetros, tiene interés morfológico y clínico.

DEL SOL, M.; OLAVE, E.; CONTRERAS, J. \& VÁSQUEZ, B. Morphometry of the sciatic, tibial and common fibular nerves in man. Int. J. Morphol., 28(2):385-388, 2010.

SUMMARY: The sciatic nerve, the longest of the human body, emerging from the pelvis through the greater sciatic foramen, dividing proximal to the knee joint in the tibial and common fibular nerves. Frequently these nerves are affected by accidents, therefore, the repairing microsurgery requires detailed anatomical information in order to successfully complete these procedures. The sciatic nerve can be damaged by penetrating injuries in the posterior luxations of the hip joint, and by intramuscular injections, given incorrectly the gluteal region. Injury to the tibial nerve is rare as it is deeply located and protected. On the other hand, injury to the common fibular nerve is frequent due to its superficial position, leaving it exposed when it leaves the popliteal fossa and surrounds the fibular neck. The study was conducted due to scarce morphometric and stereologic information on these nerves. The sciatic tibial and common fibular nerves in five cadavers of individual male adults were dissected. Transverse section of each of the nerves were obtained at the level of the division of the sciatic nerve and of the origin of the tibial and common fibular nerves. Sections of $5 \mu \mathrm{m}$ were cut which were stained with H.E. We obtained quantitative information regarding morphomtric measurements, determined the number of nerve fibres of each fascicle, as well as the number of existing fascicles in each nerve. We also determined by planimetry, through counting of points, the area of the fascicles that constituted each nerve. The number of fascicles in the sciatic nerve varied from 63 to 70 with an average of 66.8 (S.D. 2.59). In the tibial nerve the number of fascicles varied from 17 to 25 with an average of 21 (S.D. 2.92). The mean number of fibres in the sciatic nerve as of 64,535 (S.D. 3,193). In the tibial nerve it was 40,317 (S.D. 4,067) and in the common fibular nerve it was: 22,191 (S.D. 1,038). The median area of the sciatic, tibial and common fibular nerves was: $11,42 \mathrm{~mm}^{2}$ (S.D. 0,54), $8.27 \mathrm{~mm}^{2}$ (S.D. 0.69) y $3.71 \mathrm{~mm}^{2}$ (S.D. 0.30 ), respectively. With this study we hope to contribute to morphometric knowledge of these nerves, thereby providing anatomical as well as surgical support.

KEY WORDS: Sciatic nerve; Tibial nerve; Common fibular nerve; Morphometry.

\section{REFERENCIAS BIBLIOGRÁFICAS}

Del Sol, M. Vasconcellos, A.; Parra, R. \& Vásquez, B. Morfometría de los nervios tibial y plantares. Int. J. Morphol., 23(4):399-404, 2005.

Del Sol, M. Vásquez, N. \& Nicklas, C. Morfometría de los ramos del nervio plantar lateral antes de su división en ramos superficial y profundo. Int. J. Morphol., 24(1):1914, 2006.

Llano, E. G.; Flores Quintana, C. I.; Cabrera, W. R. \& Báez, A. D. Conformación fascicular de los nervios tibial y peroneo común en el perro. Rev. vet., 19(2):135-8, 2008.

Mandarim-de-Lacerda, C. A. Métodos quantitativos em morfologia. Rio de Janeiro, EDUERJ, 1995.

Moore, K. L. \& Dalley, A. F. Anatomía con orientación clínica. 4a . ed. Buenos Aires, Panamericana, 2002.
Orts, L. F. Anatomía humana. Barcelona, Científico Médica, 1982.

Testut, L. \& Jacob, O. Tratado de anatomía topográfica con aplicaciones médico quirúrgicas. $8^{\mathrm{a}}$. ed. Barcelona, Salvat., 1978.

Testut, L. \& Latarjet, A. Tratado de anatomía humana. Barcelona, Salvat, 1969.

Williams, P. L.; Warwick, R.; Dyson, M. \& Bannister, L. H. Gray Anatomia. 37 a ed. Rio de Janeiro, Guanabara\& Koogan, 1995.

Dirección para correspondencia:

Prof. Dr. Mariano del Sol

Facultad de Medicina

Universidad de La Frontera

Casilla 54-D

Temuco - CHILE

Email: mdelsol@ufro.cl
Recibido : 17-11-2009

Aceptado: 24-03-2010 